\title{
Follow-up on the therapeutic effects of a budesonide, azithromycin, montelukast, and acetylcysteine (BAMA) regimen in children with post-infectious bronchiolitis obliterans
}

\author{
Tingting Weng ${ }^{1}$, Xixi Lin ${ }^{2}$, Leying Wang ${ }^{1}$, Jiamei Lv ${ }^{1}$, Lin Dong ${ }^{1}$ \\ ${ }^{1}$ Department of Pediatrics, The Second Affiliated Hospital and Yuying Children's Hospital of Wenzhou Medical University, Wenzhou, China; \\ ${ }^{2}$ Department of Pharmacy, The Second Affiliated Hospital and Yuying Children's Hospital of Wenzhou Medical University, Wenzhou, China \\ Contributions: (I) Conception and design: L Dong; (II) Administrative support: L Dong, X Lin; (III) Provision of study materials or patients: T Weng, \\ L Wang, J Lv; (IV) Collection and assembly of data: T Weng, X Lin; (V) Data analysis and interpretation: T Weng, L Wang, J Lv; (VI) Manuscript \\ writing: All authors; (VII) Final approval of manuscript: All authors. \\ Correspondence to: Lin Dong. Department of Pediatrics, The Second Affiliated Hospital and Yuying Children's Hospital of Wenzhou Medical \\ University, 109 Xueyuan Western Road, Wenzhou, China. Email: donglinek@163.com.
}

Background: Post-infectious bronchiolitis obliterans (PIBO) is a rare, severe chronic lung disease without
optimal treatment. Currently, treatment in children mainly relies on systemic corticosteroids, but long-term
use of these drugs may lead to adverse reactions. This study aimed to evaluate the short-term efficacy of the
budesonide, azithromycin, montelukast, and acetylcysteine (BAMA) regimen in paediatric PIBO patients and
whether it can reduce systemic corticosteroid use.

Methods: This was a prospective study. From June 2017 to July 2020, patients diagnosed with PIBO at Yuying Children's Hospital of Wenzhou Medical University were treated with the BAMA regimen for 3 months. Methylprednisolone was added only when the clinical manifestations did not improve or deteriorated. All patients were followed up every 2 to 4 weeks, and changes in clinical symptoms were recorded. Pulmonary function tests and high-resolution computed tomography (HRCT) were performed, and systemic corticosteroid use was recorded after the 3-month follow-up.

Results: A total of 75 patients with PIBO were included; overall, 54 patients completed the course of treatment. After treatment, the respiratory manifestations were improved in 37 patients $(68.5 \%)$, but 4 patients $(7.4 \%)$ showed decreased exercise tolerance, and 2 patients $(3.7 \%)$ were readmitted to the hospital. Additionally, 17 (56.7\%) of the 30 patients whose pulmonary function was re-examined showed improvement, and $28(77.8 \%)$ of the 36 patients who underwent HRCT showed marked improvement. Importantly, 20 patients $(54.1 \%)$ received systemic corticosteroids for 2 weeks or less, while $31.5 \%$ of patients used no corticosteroids.

Conclusions: The BAMA regimen effectively relieved clinical symptoms and signs of PIBO in children, improved pulmonary function and HRCT manifestations, and reduced the use of systemic corticosteroids.

Keywords: Post-infectious bronchiolitis obliterans (PIBO); budesonide; azithromycin; montelukast; acetylcysteine

Submitted Oct 30, 2020. Accepted for publication Jul 14, 2021.

doi: $10.21037 /$ jtd-20-3195

View this article at: https://dx.doi.org/10.21037/jtd-20-3195

\section{Introduction}

Bronchiolitis obliterans (BO) is a rare chronic lung disease that can cause irreversible obstruction or occlusion of small airways and mainly results from a severe insult to the lower respiratory tract (1). There have been many reports of BO developing after bone marrow transplantation (BMT), haematopoietic stem cell transplantation (HSCT), or lung transplantation (2-7). Additionally, the 
occurrence of $\mathrm{BO}$ in the context of autoimmune diseases has been reported (8). In children, the most common type of $\mathrm{BO}$ is post-infectious bronchiolitis obliterans (PIBO) (9). Pathogens such as adenovirus (ADV), respiratory syncytial viruses (RSV), and influenza viruses (IV) can seriously damage the lower airways, causing inflammation and fibrosis of the terminal bronchioles and respiratory bronchioles, leading to tracheal stenosis and/ or complete occlusion after lower respiratory tract injury (9-12).

Although histopathology is still the gold standard for diagnosing and classifying PIBO, the procedure cannot be performed routinely owing to its invasive nature $(9,13)$. Consequently, many experts diagnose PIBO on the basis of the clinical course, lung function, and characteristic computed tomography (CT) findings $(10,11,14)$.

PIBO seriously worsens the quality of life of affected children (15). At present, there is no accepted best treatment for children with PIBO, who mainly receive anti-inflammatory treatment. The use of systemic glucocorticoids has been proposed as a preferred method of PIBO treatment, but intravenous methylprednisolone requires frequent hospitalization during treatment, and the incidences of side effects and complications from this approach are high $(16,17)$. Thus, it has been suggested that prolonged treatment with systemic corticosteroids should be avoided in paediatric patients (16). Some studies have shown that inhaled corticosteroids (ICS) have a significant therapeutic effect on post-HSCT patients with bronchiolitis obliterans syndrome (BOS) $(18,19)$. Azithromycin is an indispensable drug for the treatment of PIBO in children because of its anti-inflammatory effects (20). Other studies have also shown that montelukast sodium and acetylcysteine have therapeutic effects in BO $(21,22)$. At present, a combination regimen is used mainly for the treatment of adult BOS, with only one domestic study reporting the use of a combination therapy for children with PIBO (23). Here, on the basis of the encouraging results from studies of the fluticasone, azithromycin, and montelukast (FAM) regimen and the budesonide/formoterol, montelukast and $\mathrm{N}$-acetylcysteine combination in the treatment of BOS after HSCT in adults, we conducted a prospective study to evaluate the efficacy of the budesonide, azithromycin, montelukast, and acetylcysteine (BAMA) regimen in paediatric patients with PIBO $(5,19)$. Furthermore, we evaluated whether the BAMA regimen could reduce systemic corticosteroid use in these patients.

We present the following article in accordance with the
STROBE reporting checklist (available at https://dx.doi. org/10.21037/jtd-20-3195).

\section{Methods}

\section{Study subjects}

Patients with recurrent respiratory symptoms such as coughing and wheezing after lower respiratory tract infection, who went to the Pediatric Respiratory Department of the Second Affiliated Hospital and Yuying Children's Hospital of Wenzhou Medical University during the period from June 2017 to July 2020 were enrolled in this study. An experienced paediatric respiratory specialist treated all patients who received a clinical diagnosis of PIBO using an identical regimen and follow-up evaluation.

The inclusion criteria were meeting PIBO's clinical diagnostic criteria and the use of the BAMA regimen for treatment. The only exclusion criterion was that the BAMA regimen was applied for less than 3 months.

The study was conducted in accordance with the Declaration of Helsinki (as revised in 2013). The study protocol was approved by our institutional review board (IRB No. LCKY2020-241). Prior to the inclusion of children in the study, the written, informed consent of their guardians was obtained.

\section{Definition of PIBO}

The diagnosis of PIBO was made according to the following diagnostic criteria $(10,11,14)$ : history of an acute and severe lower respiratory tract infection in a previously healthy child; repeated or persistent cough, wheezing, or shortness of breath over the 6 weeks after acute infection with decreased exercise tolerance and no response to bronchodilators; mosaic perfusion signs, bronchial wall thickening, bronchiectasis, or atelectasis on chest HRCT; obstructive ventilatory dysfunction on pulmonary function tests; and a lack of other obstructive pulmonary diseases, such as asthma, pulmonary tuberculosis, bronchopulmonary dysplasia, cystic fibrosis, immunodeficiency, and $\alpha-1-$ antitrypsin deficiency.

\section{BAMA regimen}

Patients who met the diagnostic criteria for PIBO received 3 months of combination therapy in the acute 
phase. Budesonide $(0.5 \mathrm{mg})$ was inhaled twice per day. Azithromycin $(5 \mathrm{mg} / \mathrm{kg})$ was administered orally once every other day. Montelukast (4 mg) was administered once daily at bedtime, and acetylcysteine was administered three times per day at the following doses stratified by age: $33 \mathrm{mg}$ (<6 months old), $50 \mathrm{mg}$ (6-12 months old) and $100 \mathrm{mg}$ ( $\geq 1$ year old). These patients were all managed by two paediatric respiratory specialists and followed up at the outpatient clinic every $2-4$ weeks for three months.

\section{Oral systemic corticosteroids}

Low-dose methylprednisolone was administered twice per day for 3-5 days at the following doses stratified by age: $4 / 3 \mathrm{mg}$ (<6 months old), $2 \mathrm{mg}$ (6-12 months old) or $4 \mathrm{mg}$ ( $\geq 1$ year old). The same dose was then administered orally once per day for 5-7 days as a course of treatment. If the clinical manifestations did not significantly improve, the above course of treatment was repeated as necessary, and if they worsened, re-hospitalized was required.

\section{Other treatment}

Appropriate education was provided by experienced clinicians, and the avoidance of second-hand smoke and other inhaled irritants and the maintenance of respiratory clearance and adequate nutritional intake were recommended. Symptomatic treatment was provided for patients whose clinical manifestations deteriorated, including the inhalation of $2.5 \mathrm{mg}$ of nebulized terbutaline twice per day for 3-7 days. When a bacterial infection occurred, amoxicillin and clavulanate potassium for suspension were administered at the following proportions once every $12 \mathrm{~h}$ for $1-2$ weeks, stratified by age: 0.33 package ( $<3$ months old), 0.5 package (6-24 months old), 1 package ( $2-7$ years old), and 1.5 packages ( $\geq 7$ years old). Long-term family oxygen therapy was used for children with symptoms of hypoxia, such as dyspnoea, cyanosis, and irritability.

\section{Data collection}

Patient general information, clinical manifestations, pulmonary function test and lung HRCT results were collected when PIBO was diagnosed. Respiratory symptoms (including cough, wheeze and dyspnoea) and any additional treatments were recorded every 2-4 weeks during followup in the outpatient clinic. The patients received tidal respiratory pulmonary function analyses ( $<3$ years old), impulse oscillometry (IOS) (3-5 years old), and routine pulmonary ventilation function tests ( $>5$ years old) to evaluate pulmonary function after 3 months of combination therapy. Spirometric parameters, ratio of time to peak tidal expiratory flow to total expiratory time (TPEF/TE), ratio of time to peak tidal expiratory flow to total expiratory volume (VPEF/VE), respiratory system resistance at $5 \mathrm{~Hz}$ (Rrs5), respiratory reactance at $5 \mathrm{~Hz}(\mathrm{Zrs} 5)$, respiratory system resistance at $20 \mathrm{~Hz}(\operatorname{Rrs} 20)$, and forced expiratory volume in 1 second/forced vital capacity (FEV1/FVC) were collected and compared. HRCT was employed to evaluate any changes in pulmonary lesions.

\section{Statistical analysis}

The statistical analysis was performed using SPSS version 19.0 for Windows. Measurement data are expressed as the mean \pm standard deviation or the median and interquartile range (IQR), and count data are expressed as percentiles. The chi-square test or Fisher's exact test was used for analysing categorical variables. The Shapiro-Wilk test was performed to check that each continuous variable had a normal distribution. The paired $t$-test was used to compare two groups. In addition, a value of $\mathrm{P}<0.05$ was considered to indicate statistical significance.

\section{Results}

\section{Study population}

A total of 75 patients met the study inclusion criteria; among them, 3 patients refused to participate because the effect of treatment was uncertain $(\mathrm{n}=2)$ or travel was inconvenient (traffic; $\mathrm{n}=1$ ), and 1 patient planned to be treated in another hosptial. Therefore, 71 patients were allocated to the combination treatment group. However, the clinical manifestations of 5 patients improved after 0.5 months of comprehensive treatment, those of 6 patients improved after 1.5 months of treatment, and those of 1 patient improved after 2.5 months of treatment. As a result, a total of 12 patients terminated the treatment spontaneously. Five patients had not completed 3 months of treatment by the study deadline. Ultimately, a total of 54 patients (40 male and 14 female) with a median age of 1.27 years (IQR, 0.88-2.4 years) completed 3 months of the combined treatment (Figure 1). The basic patient characteristics of these individuals are shown in Table 1. 


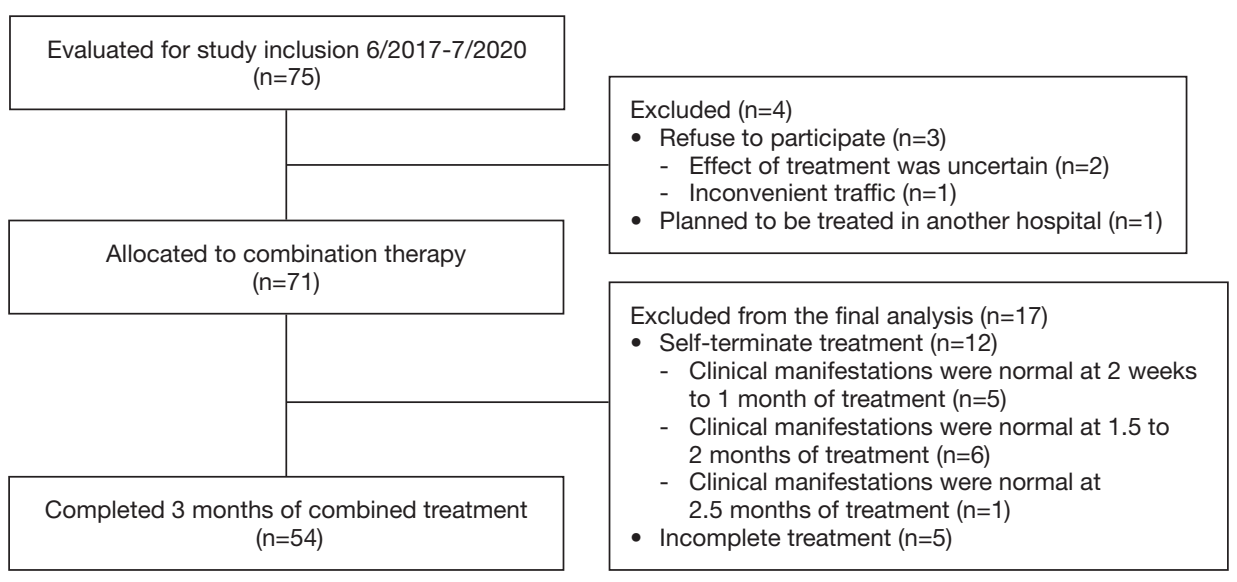

Figure 1 Flow chart of the patients with PIBO who received combination treatment. PIBO, post-infectious bronchiolitis obliterans.

Table 1 Characteristics of the patients with PIBO

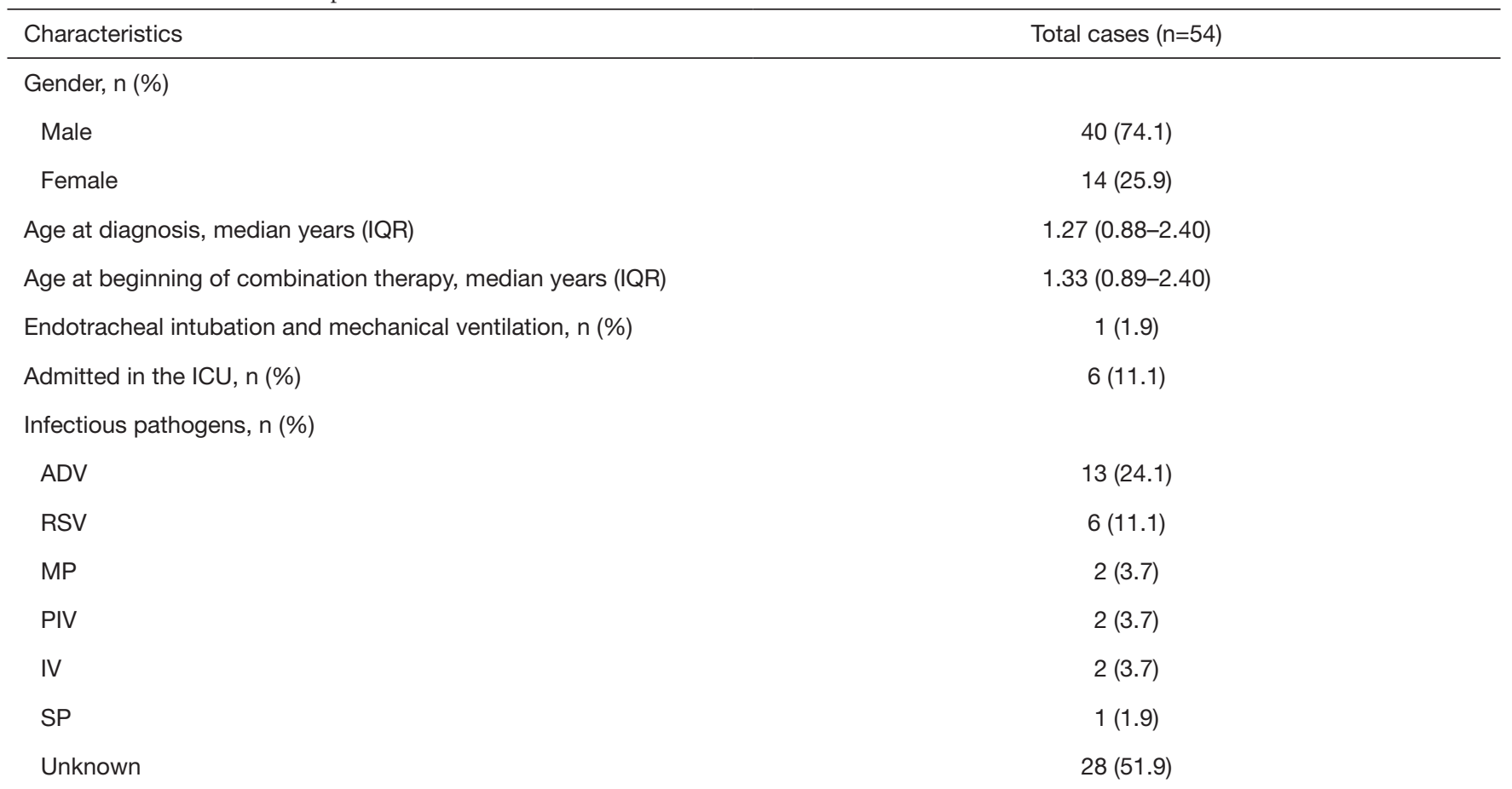

PIBO, post-infectious bronchiolitis obliterans; IQR, interquartile range; ICU, intensive care unit; ADV, adenovirus; RSV, respiratory syncytial virus; MP, Mycoplasma pneumoniae; PIV, parainfluenza virus; IV, influenza virus; SP, Streptococcus pneumoniae.

Pathogens were identified in 26 patients $(48.1 \%)$ and were not identified in 28 patients (51.9\%). ADV was the most common aetiological agent $(\mathrm{n}=13)$. Other microorganisms, including RSV, Mycoplasma pneumoniae (MP), parainfluenza virus (PIV), IV and Streptococcus pneumoniae (SP), were also detected.

\section{Systemic corticosteroids use by patients with PIBO during follow-up}

A total of 37 patients $(68.5 \%)$ used oral corticosteroids; among them, 2 patients $(5.4 \%)$ used oral corticosteroids for more than 1 month, 15 patients $(40.5 \%)$ used oral 
Table 2 Clinical manifestations of PIBO in children at diagnosis and after 3 months of BAMA treatment $(\mathrm{n}=54)$

\begin{tabular}{|c|c|c|c|c|}
\hline Clinical manifestations & PIBO diagnosis (\%) & 3 months treatment (\%) & $\chi^{2}$ & $P$ value \\
\hline Cough & $54(100.0)$ & $26(48.15)$ & - & $<0.001$ \\
\hline Wheezing & $46(85.2)$ & $9(16.7)$ & 50.72 & $<0.001$ \\
\hline Shortness of breath & $19(35.2)$ & $2(3.7)$ & 15.13 & $<0.001$ \\
\hline \multicolumn{5}{|l|}{ Sign } \\
\hline Moist rales & $48(88.9)$ & $10(18.5)$ & 53.78 & $<0.001$ \\
\hline Dry rales & $38(70.4)$ & $7(13.0)$ & 36.61 & $<0.001$ \\
\hline Three concave signs & $18(33.3)$ & $3(5.6)$ & 11.59 & 0.001 \\
\hline
\end{tabular}

PIBO, post-infectious bronchiolitis obliterans; BAMA, budesonide, azithromycin, montelukast, and acetylcysteine.

corticosteroids for 2 weeks to 1 month, and 20 patients $(54.0 \%)$ used oral corticosteroids for less than 2 weeks. The other 17 patients $(31.5 \%)$ did not use any oral corticosteroids after concluding BAMA combination therapy.

\section{Changes in clinical manifestations of PIBO after 3 months of BAMA treatment}

At the study endpoint, the clinical manifestations of PIBO were improved in 37 patients $(68.5 \%)$, unchanged in 13 patients $(24.1 \%)$, and aggravated in 4 patients $(7.4 \%)$. As indicated in Table 2, the clinical manifestations were improved significantly $(\mathrm{P}<0.05)$ after 3 months of treatment. Two patients $(3.7 \%)$ were re-hospitalized because of a severe lower respiratory infection, with one patient being readmitted once and one patient being readmitted twice. There were no obvious side effects observed during the 3 months of BAMA treatment, and only three patients developed anorexia and occasionally nausea during the follow-up period. The liver function, kidney function, and electrolyte values of the patients with PIBO were normal after receiving the combination treatment.

\section{Pulmonary function test results after 3 months of BAMA treatment}

Some patients $(n=24)$ were too young to cooperate with the examination, or had a poor sedative effect during tidal respiratory pulmonary function analyses, and did not undergo a lung function re-examination. Therefore, 30 patients $(55.6 \%)$ underwent lung function re-examination after 3 months of BAMA treatment. The results show that the pulmonary function test results improved in 17 patients but did not improve in 13 patients. At baseline, the tidal respiratory pulmonary function was low in 26 patients, with an average TPEF/TE of $19.6 \% \pm 6.1 \%$ and VPEF/ VE $23.2 \% \pm 4.9 \%$. Compared with the baseline, the average TPEF/TE $(23.2 \% \pm 8.4 \%)$ and VPEF/VE $(26.4 \% \pm 6.8 \%)$ after 3 months of treatment were improved $(\mathrm{P}<0.05)$ (Figure $2 A$ ). Among these 26 patients, 16 patients experienced improvement in their tidal respiratory pulmonary function. Three patients were examined by the use of IOS, and the Rrs5\%, Rrs20\%, Xrs5\% were not obviously improved (Figure 2B). Additionally, the FEV1/ FVC value of one patient who underwent a conventional ventilation examination increased from a baseline value of 80.8 to 99.5 after treatment (Figure 2C).

\section{Chest HRCT results after 3 months of BAMA treatment}

Chest HRCT was repeated after BAMA treatment in 36 patients. At baseline, 29 of these patients had uneven ventilation with typical mosaic signs, six had emphysema, three had bronchiectasis, two had bronchial wall thickening, and one had atelectasis (Table 3). Twenty-eight patients showed marked improvements at the combination therapy endpoint, while six patients had no changes, and two patients deteriorated. Impressively, the mosaic signs improved in $24(82.8 \%)$ patients. Among the patients 

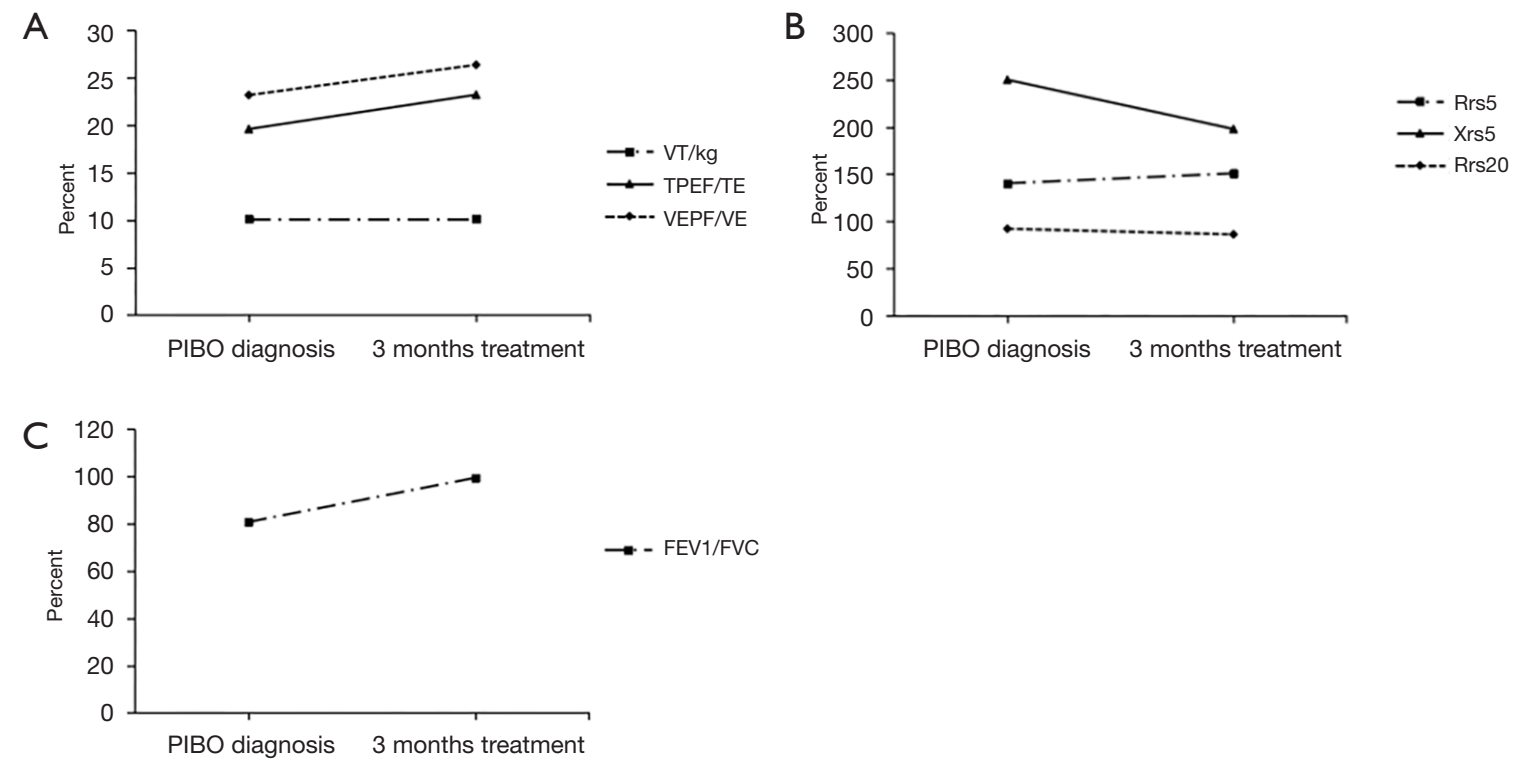

Figure 2 Changes in pulmonary function after 3 months of combination treatment. (A) After 3 months of combination treatment, the TPEF/TE and VPEF/VE values improved significantly, whereas the VT/kg did not change (n=26); (B) the Rrs5\%, Rrs20\% and Zrs5\% showed no obvious improvement after combination therapy $(n=3)$; (C) the FEV1/FVC value of one case was improved significantly. TPEF/ TE, ratio of time to peak tidal expiratory flow to total expiratory time; VPEF/VE, ratio of volume to peak tidal expiratory flow to total expiratory volume; VT/kg, tidal volume over body weight; Rrs5, respiratory system resistance at $5 \mathrm{~Hz}$; Rrs20, respiratory system resistance at $20 \mathrm{~Hz}$; Zrs5, respiratory reactance at $5 \mathrm{~Hz}$; FEV1, forced expiratory volume in 1 second; FVC, forced vital capacity.

Table 3 HRCT changes after 3 months of combination therapy $(\mathrm{n}=36)$

\begin{tabular}{|c|c|c|c|c|}
\hline Manifestations of CT imaging & PIBO diagnosis $(n)$ & \multicolumn{3}{|c|}{ Endpoints } \\
\hline Mosaic pattern & 29 & 24 & 4 & 1 \\
\hline Emphysema & 6 & 3 & 1 & 2 \\
\hline Bronchiectasis & 3 & 2 & 0 & 1 \\
\hline Atelectasis & 1 & 1 & 0 & 0 \\
\hline
\end{tabular}

HRCT, high-resolution computed tomography; CT, computed tomography; PIBO, post-infectious bronchiolitis obliterans.

with emphysema at baseline, three improved, one had no change, and two deteriorated. Moreover, significant improvement was observed in two of the three patients with bronchiectasis. Finally, the two patients with bronchial wall thickening and one patient with atelectasis also showed improvement.

\section{Discussion}

In the present study, we retrospectively evaluated and monitored the therapeutic effects of the BAMA regimen in children with PIBO. After 3 months of the combination treatment, respiratory manifestations, lung function, and HRCT manifestations were significantly improved in our cohort without obvious adverse reactions. Seventeen of 30 patients had significantly increased lung function after 3 months of combination treatment. At the end of treatment, $77.8 \%$ of the children had improved lung imaging results. These findings support the feasibility of the BAMA regimen for the treatment of PIBO in 
children. Importantly, our BAMA regimen also helped to reduce prolonged systemic oral steroid use in children with PIBO.

A few studies have confirmed airway inflammation among patients with cases of $\mathrm{BO}$ that began in childhood $(24,25)$. The levels of neutrophils, IL- 8 and CD $8^{+} \mathrm{T}$ lymphocytes were found to be elevated in BALF samples in children with PIBO $(24,25)$. Therefore, anti-inflammatory agents are currently the main treatment for children with PIBO. Corticosteroids are effective anti-inflammatory drugs that have been used to treat respiratory diseases since 1950 (26). A retrospective, uncontrolled study of 40 children with PIBO concluded that pulsed corticosteroid therapy improved lung function and safely replaced longterm oral corticosteroid therapy, minimizing adverse reactions to oral therapy (27). Continuous systemic corticosteroid therapy may be needed to maintain increased lung function and prevent recurrence of airway inflammation in this population (28). Systemic corticosteroids should be used during the development of disease and before the establishment of airway fibrosis (29). However, corticosteroids can cause serious side effects and complications, such as bone fractures or mortality from infections (16). In addition, they have a negative impact on the growth and development of children as well as on their bone metabolism; thus, reducing the use of systemic steroids for the treatment of PIBO in children is recommended (17,30,31). In our study, 20 patients $(54.1 \%)$ received corticosteroids for less than 2 weeks, and $31.5 \%$ of the patients did not use corticosteroids after receiving the BAMA regimen.

ICS have been found to have a significant therapeutic effect and reduce the side effects of systemic corticosteroid therapy in constrictive bronchiolitis after HSCT (32). A randomized, double-blind, placebo-controlled study reported that the FEV1 value after HSCT in adults with BOS increased by $13 \%$ after 1 month of budesonide/ formoterol treatment (18). Zhang et al. found that, after 12 months of treatment with inhaled budesonide combined with terbutaline and ipratropium bromide, the clinical scores and the average of TPEF/TE and VPEF/VE values of children with PIBO were significantly improved, the frequency and severity of deterioration in these children were reduced, and the HRCT results for these patients were significantly improved (33). These encouraging results provide new insights into the treatment of PIBO with ICS. However, short-term ICS had no obvious curative effect, and long-term aerosol treatment was required.
Azithromycin has been reported to have beneficial effects in BOS cases; patients who were treated with azithromycin had improved FEV1 values, and reduced airway neutrophil and IL-8 levels (34). In the study conducted by Corris et al., the FEV1 value after lung transplantation in patients with BOS was improved after 12 weeks of azithromycin treatment (35). A recent meta-analysis demonstrated that after 7 months of azithromycin treatment, the post-lung transplantation FEV1 value in patients with BOS had increased by $8.8 \%$ (36). Wang et al. treated found that the clinical and HRCT manifestations were significantly improved in 10 of 16 infants with PIBO who were treated with prednisolone and azithromycin for 6-27 months (20). Therefore, long-term ICS combined with low-dose azithromycin therapy may be a new and safe treatment for children with PIBO.

Montelukast is a leukotriene receptor antagonist (LTRA) that has anti-inflammatory effects and is generally considered a safe drug (37). Studies have shown that montelukast is well tolerated in children aged 1-6 months $(38,39)$. Animal models have demonstrated that LTRA can prevent tracheal fibrosis in BO after lung transplantation (40). In a randomized controlled trial on post-transplant $\mathrm{BO}$ in adults, montelukast attenuated further decreases in the FEV1 value in patients (41). For patients with low neutrophil percentages $(<15 \%)$ and BOS after lung transplantation who have received or are concurrently receiving azithromycin therapy, the addition of montelukast may be a promising treatment option (21).

$\mathrm{N}$-acetylcysteine is also a relatively safe and effective option for treating BO $(42,43)$. This mucolytic agent has antioxidant and anti-inflammatory effects, and it can control the symptoms of respiratory diseases and improve lung function $(44,45)$. Additionally, $\mathrm{N}$-acetylcysteine can inhibit airway thickening and bronchial smooth muscle hypertrophy by acting on bronchial smooth muscle (46). Other prospective studies have also found that the long-term use of $\mathrm{N}$-acetylcysteine can reduce the risk of exacerbations and provide a significantly improvement in the quality of life for bronchiectasis patients (47). One study found that treatment with clarithromycin and acetylcysteine together improved the symptoms and lung function of patients with $\mathrm{BO}$ who were exposed to sulphur mustard gas (42). Ghanei et al. found that respiratory tract symptoms were improved and vital capacity was significantly improved in 144 patients with BOS who were treated with acetylcysteine for 4 months (22). A 5 -year study of children with $\mathrm{BO}$ associated with mustard inhalation showed that 
azithromycin, inhaled steroids, and $\mathrm{N}$-acetylcysteine were effective at delaying the decline in FEV1 (43).

The best effective combination treatment for PIBO is unknown. The FAM regimen, which features inhalation of fluticasone, azithromycin and montelukast, can prevent a decline of $10 \%$ or greater in the FEV1 value in $72 \%$ of patients with BOS after HSCT, and $48 \%$ of patients reduced their steroid dose by $50 \%$ or more within 3 months (5). Additionally, the combination of budesonide/formoterol, montelukast, and $\mathrm{N}$-acetylcysteine significantly improved the pulmonary function and respiratory symptoms of $82 \%$ of adult patients with BOS after HSCT without serious side effects (19). However, these two studies focused on BOS after HSCT in adults and did not evaluate the changes on pulmonary HRCT in patients after 3 months of treatment. Moreover, a recent study showed that the combination of budesonide, montelukast and azithromycin can significantly improve pulmonary function and respiratory symptoms in children with PIBO who are less than 5 years old; however, simultaneous HRCT improvement after treatment was not observed (23).

We acknowledge that this study has some limitations. First, it was not a randomized controlled study, and it did not compare the efficacy of the treatment combination with that of a placebo. Second, the beneficial effects of our combination therapy on respiratory manifestations, lung function, and lung HRCT manifestations need to be confirmed in further studies evaluating each drug and their interaction. Third, the relatively short follow-up period limited the assessment of long-term prognosis, as 3 months is not long enough to cover the natural course of the disease. However, we simultaneously evaluated the lung changes on HRCT before and after treatment in children with PIBO. Well-designed methods are necessary for further research to evaluate the long-term effectiveness and safety of the BAMA combination treatment.

\section{Conclusions}

This is a real-world single-centre experience for PIBO treatment in a children's hospital in China. The results suggest that the BAMA regimen can improve the respiratory manifestations, pulmonary function, and uneven pulmonary ventilation and can reduce oral systemic corticosteroid use; thus, it is of great significance for the clinical treatment of children with PIBO.

\section{Acknowledgments}

Funding: None.

\section{Footnote}

Reporting Checklist: The authors have completed the STROBE reporting checklist. Available at https://dx.doi. org/10.21037/jtd-20-3195

Data Sharing Statement: Available at https://dx.doi. org/10.21037/jtd-20-3195

Conflicts of Interest: All authors have completed the ICMJE uniform disclosure form (available at https://dx.doi. org/10.21037/jtd-20-3195). The authors have no conflicts of interest to declare.

Ethical Statement: The authors are accountable for all aspects of the work in ensuring that questions related to the accuracy or integrity of any part of the work are appropriately investigated and resolved. The study was conducted in accordance with the Declaration of Helsinki (as revised in 2013). This study was approved by the Medical Ethics Committee of the second affiliated Hospital of Wenzhou Medical University, under the registration number of LCKY2020-241.

Open Access Statement: This is an Open Access article distributed in accordance with the Creative Commons Attribution-NonCommercial-NoDerivs 4.0 International License (CC BY-NC-ND 4.0), which permits the noncommercial replication and distribution of the article with the strict proviso that no changes or edits are made and the original work is properly cited (including links to both the formal publication through the relevant DOI and the license). See: https://creativecommons.org/licenses/by-nc-nd/4.0/.

\section{References}

1. Hardy KA, Schidlow DV, Zaeri N. Obliterative bronchiolitis in children. Chest 1988;93:460-6.

2. Ooi GC, Peh WC, Ip M. High-resolution computed tomography of bronchiolitis obliterans syndrome after bone marrow transplantation. Respiration 1998;65:187-91.

3. Jung JI, Jung WS, Hahn ST, et al. Bronchiolitis obliterans 
after allogenic bone marrow transplantation: HRCT findings. Korean J Radiol 2004;5:107-13.

4. Ratjen F, Rjabko O, Kremens B. High-dose corticosteroid therapy for bronchiolitis obliterans after bone marrow transplantation in children. Bone Marrow Transplant 2005;36:135-8.

5. Williams KM, Cheng GS, Pusic I, et al. Fluticasone, Azithromycin, and Montelukast Treatment for New-Onset Bronchiolitis Obliterans Syndrome after Hematopoietic Cell Transplantation. Biol Blood Marrow Transplant 2016;22:710-6.

6. Walther S, Rettinger E, Maurer HM, et al. Long-term pulmonary function testing in pediatric bronchiolitis obliterans syndrome after hematopoietic stem cell transplantation. Pediatr Pulmonol 2020;55:1725-35.

7. Miyagawa-Hayashino A, Wain JC, Mark EJ. Lung transplantation biopsy specimens with bronchiolitis obliterans or bronchiolitis obliterans organizing pneumonia due to aspiration. Arch Pathol Lab Med 2005;129:223-6.

8. Kanaoka M, Matsukura S, Ishikawa H, et al. Paraneoplastic pemphigus associated with fatal bronchiolitis obliterans and appearance of anti-BP180 antibodies in the late stage of the disease. J Dermatol 2014;41:628-30.

9. Kavaliunaite E, Aurora P. Diagnosing and managing bronchiolitis obliterans in children. Expert Rev Respir Med 2019;13:481-8.

10. Li YN, Liu L, Qiao HM, et al. Post-infectious bronchiolitis obliterans in children: a review of 42 cases. BMC Pediatr 2014;14:238.

11. Fischer GB, Sarria EE, Mattiello R, et al. Post infectious bronchiolitis obliterans in children. Paediatr Respir Rev 2010;11:233-9.

12. Murtagh P, Giubergia V, Viale D, et al. Lower respiratory infections by adenovirus in children. Clinical features and risk factors for bronchiolitis obliterans and mortality. Pediatr Pulmonol 2009;44:450-6.

13. Zhang L, Irion K, Kozakewich H, et al. Clinical course of postinfectious bronchiolitis obliterans. Pediatr Pulmonol 2000;29:341-50.

14. Colom AJ, Teper AM. Post-infectious bronchiolitis obliterans. Pediatr Pulmonol 2019;54:212-9.

15. Lee E, Shim JY, Kim HY, et al. Clinical characteristics and etiologies of bronchiectasis in Korean children: A multicenter retrospective study. Respir Med 2019;150:8-14.

16. Jerkic SP, Brinkmann F, Calder A, et al. Postinfectious Bronchiolitis Obliterans in Children: Diagnostic Workup and Therapeutic Options: A Workshop Report. Can
Respir J 2020;2020:5852827.

17. Yoon HM, Lee JS, Hwang JY, et al. Post-infectious bronchiolitis obliterans in children: CT features that predict responsiveness to pulse methylprednisolone. Br J Radiol 2015;88:20140478.

18. Bergeron A, Chevret S, Chagnon K, et al. Budesonide/ Formoterol for bronchiolitis obliterans after hematopoietic stem cell transplantation. Am J Respir Crit Care Med 2015;191:1242-9.

19. Kim SW, Rhee CK, Kim YJ, et al. Therapeutic effect of budesonide/formoterol, montelukast and N-acetylcysteine for bronchiolitis obliterans syndrome after hematopoietic stem cell transplantation. Respir Res 2016;17:63.

20. Wang X, Liu C, Wang M, et al. Clinical features of postinfectious bronchiolitis obliterans in children undergoing long-term azithromycin treatment. Exp Ther Med 2015;9:2379-83.

21. Verleden GM, Verleden SE, Vos R, et al. Montelukast for bronchiolitis obliterans syndrome after lung transplantation: a pilot study. Transpl Int 2011;24:651-6.

22. Ghanei M, Shohrati M, Jafari M, et al. N-acetylcysteine improves the clinical conditions of mustard gas-exposed patients with normal pulmonary function test. Basic Clin Pharmacol Toxicol 2008;103:428-32.

23. Chen X, Shu JH, Huang Y, et al. Therapeutic effect of budesonide, montelukast and azithromycin on postinfectious bronchiolitis obliterans in children. Exp Ther Med 2020;20:2649-56.

24. Cazzato S, Poletti V, Bernardi F, et al. Airway inflammation and lung function decline in childhood post-infectious bronchiolitis obliterans. Pediatr Pulmonol 2008;43:381-90.

25. Koh YY, Jung DE, Koh JY, et al. Bronchoalveolar cellularity and interleukin-8 levels in measles bronchiolitis obliterans. Chest 2007;131:1454-60.

26. de Benedictis FM, Canny GJ, Levison H. The role of corticosteroids in respiratory diseases of children. Pediatr Pulmonol 1996;22:44-57.

27. Tomikawa SO, Adde FV, da Silva Filho LV, et al. Followup on pediatric patients with bronchiolitis obliterans treated with corticosteroid pulse therapy. Orphanet J Rare Dis 2014;9:128.

28. Tanou K, Xaidara A, Kaditis AG. Efficacy of pulse methylprednisolone in a pediatric case of postinfectious bronchiolitis obliterans. Pediatr Pulmonol 2015;50:E13-6.

29. Moonnumakal SP, Fan LL. Bronchiolitis obliterans in children. Curr Opin Pediatr 2008;20:272-8.

30. Anthracopoulos MB, Papadimitriou A, Panagiotakos 
DB, et al. Growth deceleration of children on inhaled corticosteroids is compensated for after the first 12 months of treatment. Pediatr Pulmonol 2007;42:465-70.

31. Adinoff AD, Hollister JR. Steroid-induced fractures and bone loss in patients with asthma. N Engl J Med 1983;309:265-8.

32. Bashoura L, Gupta S, Jain A, et al. Inhaled corticosteroids stabilize constrictive bronchiolitis after hematopoietic stem cell transplantation. Bone Marrow Transplant 2008;41:63-7.

33. Zhang XM, Lu AZ, Yang HW, et al. Clinical features of postinfectious bronchiolitis obliterans in children undergoing long-term nebulization treatment. World J Pediatr 2018;14:498-503.

34. Verleden GM, Vanaudenaerde BM, Dupont LJ, et al. Azithromycin reduces airway neutrophilia and interleukin-8 in patients with bronchiolitis obliterans syndrome. Am J Respir Crit Care Med 2006;174:566-70.

35. Corris PA, Ryan VA, Small T, et al. A randomised controlled trial of azithromycin therapy in bronchiolitis obliterans syndrome (BOS) post lung transplantation. Thorax 2015;70:442-50.

36. Kingah PL, Muma G, Soubani A. Azithromycin improves lung function in patients with post-lung transplant bronchiolitis obliterans syndrome: a meta-analysis. Clin Transplant 2014;28:906-10.

37. Ramsay CF, Sullivan P, Gizycki M, et al. Montelukast and bronchial inflammation in asthma: a randomised, double-blind placebo-controlled trial. Respir Med 2009;103:995-1003.

38. Kearns GL, Lu S, Maganti L, et al. Pharmacokinetics and safety of montelukast oral granules in children 1 to 3 months of age with bronchiolitis. J Clin Pharmacol 2008;48:502-11.

39. Bisgaard H, Flores-Nunez A, Goh A, et al. Study of

Cite this article as: Weng $\mathrm{T}$, Lin $\mathrm{X}$, Wang $\mathrm{L}, \mathrm{Lv} \mathrm{J}$, Dong L. Follow-up on the therapeutic effects of a budesonide, azithromycin, montelukast, and acetylcysteine (BAMA) regimen in children with post-infectious bronchiolitis obliterans. J Thorac Dis 2021;13(8):4775-4784. doi: 10.21037/jtd-20-3195 montelukast for the treatment of respiratory symptoms of post-respiratory syncytial virus bronchiolitis in children. Am J Respir Crit Care Med 2008;178:854-60.

40. Tu ZL, Zhou ZY, Xu HC, et al. LTB4 and montelukast in transplantation-related bronchiolitis obliterans in rats. J Cardiothorac Surg 2017;12:43.

41. Ruttens D, Verleden SE, Demeyer H, et al. Montelukast for bronchiolitis obliterans syndrome after lung transplantation: A randomized controlled trial. PLoS One 2018;13:e0193564.

42. Wuyts WA, Vanaudenaerde BM, Dupont LJ, et al. $\mathrm{N}$-acetylcysteine inhibits interleukin-17-induced interleukin-8 production from human airway smooth muscle cells: a possible role for anti-oxidative treatment in chronic lung rejection? J Heart Lung Transplant 2004;23:122-7.

43. Abtahi H, Peiman S, Foroumandi M, et al. Long Term Follow-Up of Sulfur Mustard Related Bronchiolitis Obliterans Treatment. Acta Med Iran 2016;54:605-9.

44. Kupczyk M, Kuna P. Mucolytics in acute and chronic respiratory tract disorders. II. Uses for treatment and antioxidant properties. Pol Merkur Lekarski 2002;12:248-52.

45. Askari M, Faryabi R, Mozaffari H, et al. The effects of N-Acetylcysteine on serum level of inflammatory biomarkers in adults. Findings from a systematic review and meta-analysis of randomized clinical trials. Cytokine 2020;135:155239.

46. Hayashi K, Takahata H, Kitagawa N, et al. $\mathrm{N}$-acetylcysteine inhibited nuclear factor-kappaB expression and the intimal hyperplasia in rat carotid arterial injury. Neurol Res 2001;23:731-8.

47. Qi Q, Ailiyaer Y, Liu R, et al. Effect of N-acetylcysteine on exacerbations of bronchiectasis (BENE): a randomized controlled trial. Respir Res 2019;20:73. 